\title{
Empfehlungen für körperliche Aktivität und Inaktivität von Kindern und Jugendlichen - Methodisches Vorgehen, Datenbasis und Begründung
}

\author{
Recommendations for Physical Activity and Sedentary Behaviour \\ for Children and Adolescents: Methods, Database and Rationale
}

\author{
Autoren \\ Christine Graf' 1 , Nina Ferrari², Ralph Beneke ${ }^{3}$, Wilhelm Bloch ${ }^{4}$, Stefanie \\ Eiser $^{5}$, Benjamin Koch ${ }^{6}$, Wolfgang Lawrenz ${ }^{7}$, Susanne Krug ${ }^{8}$, Kristin \\ Manz ${ }^{9}$, Renate Oberhoffer ${ }^{10}$, Günter Stibbe ${ }^{11}$, Alexander Woll ${ }^{12}$ \\ Institute \\ 1 Deutsche Sporthochschule Köln, Institut für Bewegungs- und \\ Neurowissenschaft, Köln \\ 2 Kölner Zentrum für Prävention im Kindes- und Jugendalter, \\ Herzzentrum Uniklinik Köln, Köln \\ 3 Training und Gesundheit, Philipps-Universität Marburg, Bereich \\ Medizin, Marburg \\ 4 Deutsche Sporthochschule Köln, Institut für Kreislaufforschung \\ und Sportmedizin, Köln \\ 5 Bundesanstalt für Landwirtschaft und Ernährung, BLE, Bonn \\ 6 Zentrum für Adipositasschulung Bremen-Stadt e.V., ZABS e.V., \\ Bremen \\ 7 Deutsche Gesellschaft für Sportmedizin und Prävention, Ständige \\ Kommission Kinder- und Jugendsport, Frankfurt \\ 8 Robert Koch Institut, Berlin \\ 9 Robert Koch Institut, Epidemiology and Health Monitoring, Berlin \\ 10 Technische Universität München, Präventive Pädiatrie, München \\ 11 Deutsche Sporthochschule Köln, Institut für Schulsport und \\ Schulentwicklung, Köln \\ 12 Karlsruher Institut für Technologie, Sportwissenschaft, Karlsruhe
}

Schlüsselwörter

körperliche Aktivität, Empfehlungen, Inaktivität, Kinder, Jugendliche

Key words

physical activity, recommendations, sedentary behaviour, children, youth

\section{Bibliografie}

DOI http://dx.doi.org/10.1055/s-0042-123701

Gesundheitswesen 2017; 79 (Suppl. 1): S11-S19

(c) Georg Thieme Verlag KG Stuttgart · New York

ISSN 0949-7013
Korrespondenzadresse

Prof. Christine Graf

Institut für Bewegungs- und Neurowissenschaft

Deutsche Sporthochschule Köln

Am Sportpark Müngersdorf

50933 Köln

C.Graf@dshs-koeln.de

$\Theta$

Die zitierte Literatur finden Sie Online unter http://dx.doi. org/10.1055/s-0042-123701.

\section{ZUSAMMENFASSUNG}

Hintergrund Der Nutzen von körperlicher Aktivität bzw. einer Reduktion von vermeidbarer Sitzzeit ist im Kindes- und Jugendalter unbestritten. Methode Auf Basis einer qualitätsgesicherten Literaturrecherche wurden die Empfehlungen für Deutschland aktualisiert und erweitert. Sie umfassen nun dezidiert das Säuglings-, Kleinkind-, Kindergarten- und Grundschulalter sowie Jugendliche.

Ergebnisse und Schlussfolgerung Generell gilt, je mehr Bewegung desto höher der gesundheitliche Benefit. Ab dem Kindergartenalter sollten mindestens 180, ab Grundschulalter mind. 90 min Bewegungszeit pro Tag erreicht werden. Vermeidbare Sitzzeiten, insbesondere Bildschirmmedien, sollen dagegen auf ein Minimum reduziert werden.

\section{ABSTRACT}

Background The benefits of physical activity and a reduction of sedentary behaviour in childhood and adolescence are well established. Methods Based on a quality-assured literature review, the German recommendations were updated. Guidelines for infants, toddlers, children of kindergarten and primary school age and youth are clearly depicted. Results and Conclusion In general, a higher amount of physical activity is associated with more health benefits. Preschool age children should have 180 min of physical activity daily, and from primary school age on, at least 90 min daily are recommended. Sedentary behaviour, especially time in front of screens, is to be reduced to a minimum.

\section{Einleitung}

Der Nutzen von körperlicher Aktivität bzw. einer Reduktion von vermeidbarer Sitzzeit ist im Kindes- und Jugendalter unbestritten [1]. So kommt Bewegung für die körperliche, geistige, emotionale und psychosoziale Entwicklung - vor allem für jüngere Kinder eine zentrale Bedeutung zu [2,3]. Wie groß allerdings Umfänge, Dauer, Intensität und Art sein müssen und welche möglichen Unterschiede sich für die diversen Altersstufen ergeben, kann bislang noch nicht endgültig beantwortet werden. In einem Expertenkonsens im Jahr 2012 wurden für die gesamte Periode des Kindes- und Jugendalter Empfehlungen bzgl. Aktivität und Inaktivität zusammengestellt $[1,4]$. Diese wurden nun auf Basis einer systematischen Literaturrecherche der internationalen Empfehlungen bzw. systematischen Reviews seit 2010 unter Berücksichtigung der Phasen:

- Säuglinge (bis Ende des ersten Lebensjahres) und Kleinkinder (1-3 Jahre)

- Kindergartenkinder (4-6 Jahre) 
- Grundschulkinder (6-11 Jahre) und

- Jugendliche (12-18 Jahre) aktualisiert.

\section{Methodik}

Diese Empfehlungen für gesundheitswirksame körperliche Aktivität wurden auf Basis bereits bestehender, qualitativ hochwertiger Empfehlungen in einem 3-phasigen Vorgehen entwickelt (vgl. auch Beitrag von Geidl et al. 2016 in diesem Band). In Phase 1 wurden vorhandene Bewegungsempfehlungen für die jeweiligen Altersgruppen jeweils separat systematisch recherchiert. Für Kinder und Jugendliche erfolgte die Medlinerecherche nach folgenden Suchbegriffen über den Zeitraum Januar 2010 bis September 2015: (((“recommendations"[All Fields]) AND (("physical activity”[All Fields]) OR “exercise”[MeSH Terms]) AND ((“infant”[MeSH Terms] OR “child”[MeSH Terms]) OR “adolescent”[MeSH Terms])) AND ((Guideline[ptyp] OR Practice Guideline[ptyp] OR systematic[sb]) AND (“2010/01/01”[PDat]: “2015/12/31”[PDat]) AND (English[lang] OR German[lang])). Der Zeitraum 2010 wurde im Konsens in der Arbeitsgruppe festgelegt, da diesem Jahr die letzte Veröffentlichung der Weltgesundheitsorganisation entstammte [15], s.a. hierzu Beitrag Geidl et al. (2016).

In $>$ Tab. 1 wurden die bislang vorliegenden Bewegungsempfehlungen im Bereich „Kinder und Jugendliche“ zusammenfassend dargestellt. Diese wurden nach dem im Beitrag von Geidl et al. 2016 vorgestellten Verfahren einer Bewertung mit definierten Qualitätskriterien unterzogen; diese 28 Items basieren im Wesentlichen auf der DELBI- bzw. AGREE II-Methodik zur Leitlinienbewertung, die mithilfe einer modifizierten Delphi-Befragung mit nationalen Expertinnen und Experten abgestimmt und in 4 Domänen zusammengefasst wurden (Phase 2): A „Geltungsbereich und Zweck“ (3 Items), B „Methodologische Exaktheit der Entwicklung der Empfehlung“ (10 Items), C „Inhaltliche Klarheit und Differenziertheit“ (12 Items) und D „Gestaltung“ ( 3 Items). Eine Punktzahl von $\geq 60 \%$ der erzielbaren Gesamtpunkte in einer Domäne wurde jeweils als Cut-Off-Wert für eine hochwertige festgelegt wurden und bildeten damit die Basis für die Entwicklung der nationalen Empfehlungen für gesundheitswirksame Bewegung für die beschriebenen Zielgruppen; hier Kinder und Jugendliche (Phase 3; • Tab. 2).

\section{Ergebnisse}

\section{Recherche}

Die Suche ergab zunächst 58 Treffer. Aus diesen wurden Empfehlungen aus dem Kontext spezifischer Erkrankungen, wie Asthma, Zerebralparese, Diabetes, Vitien usw., sowie Sport/Bewegung in ausgewiesenen Präventions-/Therapieprogrammen (meist Adipositas) und Hinweise zu Bewegungsempfehlungen in Settings wie Schule oder Kindergärten ausgeschlossen. Damit reduzierten sich die Treffer der letzten 5 Jahre auf 4 relevante Publikationen [5-8]; eine fünfte war eine nochmalige Zusammenfassung der kanadischen Empfehlungen [9]. 3 zusätzliche Reviews wurden ergänzt und dem gleichen oben beschriebenen Beurteilungsverfahren unterzogen. Es handelte sich dabei um relevante weitere Inhalte wie Alltagsaktivitäten im Kindes- und Jugendalter (Schritte/Tag; [10]), den bislang vorliegende Expertenkonsens aus Deutschland [1] sowie ein systematisches Review über alle europäischen Empfehlungen bis Sommer 2012 [11].

Die daraus abgeleiteten Empfehlungen wurden in > Tab. 2 zusammengefasst. Sämtliche weiteren verfügbaren Empfehlungen bzw. deren Kernaussagen sowie - wenn angegeben - Änderungen, die sich in Updates im Vergleich zu zuvor veröffentlichten Empfehlungen ergaben, wurden in $>$ Tab. 1 zusammengefasst (mod. nach Kahlmeier et al. [11]). Dabei wurde die Grundstruktur von Kahlmeier et al. [11] beibehalten, die darin enthaltenen gesundheitlichen Hinweise komprimiert und um die Updates bzw. neuere Empfehlungen ergänzt.

\section{Entwicklung}

Ende der 90er Jahre wurde die empfohlene Bewegungszeit von 20 bis $30 \mathrm{~min} /$ Tag in den $80 \mathrm{er}$ Jahren auf zunächst $30 \mathrm{~min} /$ Tag festgelegt (zusammengefasst in [12]), dann auf der Basis von Expertenempfehlungen auf $60 \mathrm{~min} /$ Tag verdoppelt [13]; eine entsprechende Anpassung der WHO-Empfehlungen erfolgte im Jahr 2002 („Why Move for Health“, http://www.who.int/moveforhealth/en/ in [14]). In den aktuell zusammengefassten Empfehlungen werden überwiegend in Anlehnung an die WHO [15] 60 min körperliche Aktivität pro Tag meist moderater bis höherer Intensität empfohlen. Damit wird größtenteils der Hinweis verbunden, dass es sich um ein Minimum handelt und ein „mehr“ an Bewegung auch zu einem höheren gesundheitlichen Nutzen führt. Die wirkliche Studienlage jedoch, auf der die meisten Empfehlungen basieren, ist je nach Altersgruppe inkonsistent. Im folgenden werden die relevanten Ergebnisse bzgl. Gesundheit für die Perioden Säuglings-, Kleinkind- und Vorschulalter bzw. Grundschul- und Jugendalter gemeinsam dargestellt. Anschließend werden ausgewählte Aspekte wie Bewegungsformen in Alltag und Freizeit, mögliche Risiken sowie bezogen auf sitzende Tätigkeiten zusammengefasst.

\section{Säuglings- und Kleinkindalter bzw. Vorschulalter}

Bislang gibt es nur wenige Daten über mögliche Zusammenhänge zwischen Bewegung und Gesundheit in dieser Altersgruppe. Hier legen im Wesentlichen die angloamerikanischen Arbeitsgruppen konkrete Bewegungszeiten vor (u. a. [5-8]. So soll im Säuglingsalter die Bewegungszeit so wenig wie möglich eingeschränkt werden, im Kleinkind bzw. Kindergartenalter werden 180 min Bewegungszeit pro Tag - angeleitet und nicht angeleitet - empfohlen. Grundlage für diese Empfehlungen stellte ein systematischer Review von Timmons et al. [2] zum Zusammenhang zwischen körperlicher Aktivität und Gesundheit bei 0 - bis 4-jährigen Kindern dar. Folgende Indikatoren wurden darin untersucht: Auftreten von Übergewicht und Adipositas bzw. der Körperkomposition, kardiovaskuläre Faktoren (u. a. Blutdruck, Lipide, Glukose und Insulin sowie Entzündungsparameter), Bewegungsapparat, motorische Entwicklung, psychosoziale Gesundheit (Selbstkonzept, Selbstbewusstsein, Aggressivität, Verhalten usw.) und kognitive Entwicklung (u. a. Sprachentwicklung). Die Evidenzlage bewegte sich zwischen niedrig bis hoch, zeigte aber, dass eine Steigerung bzw. ein höheres Ausmaß an Bewegungszeit mit einer geringeren Adipositasprävalenz, weniger kardiometabolischen Risikofaktoren, höherer motorischer Leistungsfähigkeit, besserer Knochengesundheit, günstigeren psychosozialen Aspekten und höherer kognitiver Ent- 


\begin{tabular}{|c|c|c|c|c|c|c|c|c|c|}
\hline 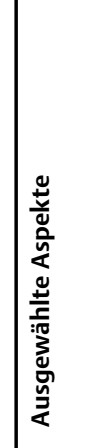 & 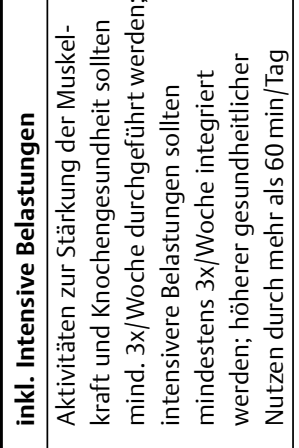 & & 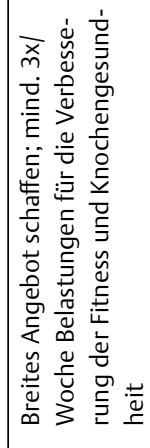 & 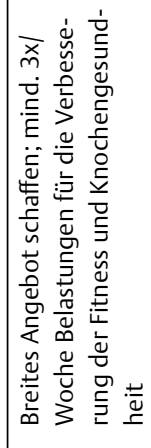 & 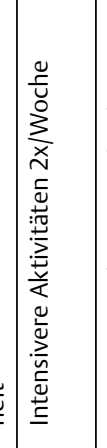 & 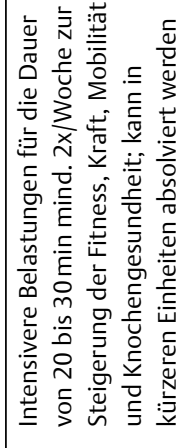 & 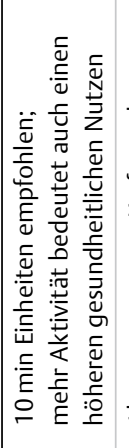 & 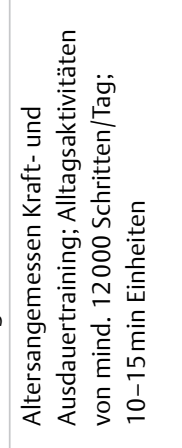 & \\
\hline $\mathbf{\sigma}$ & 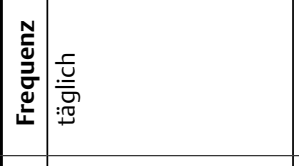 & 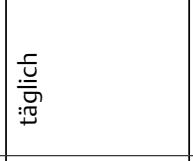 & 啮 & 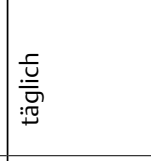 & 䇏 & 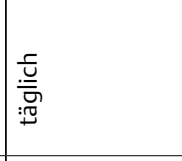 & 离 & $\frac{\frac{5}{5}}{\frac{5}{9}}$ & \\
\hline | & 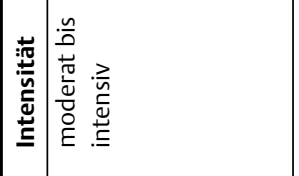 & 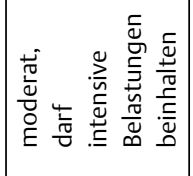 & 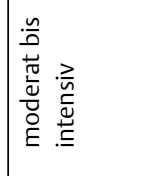 & 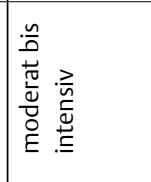 & 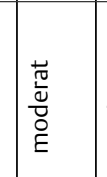 & 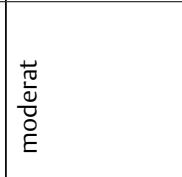 & 墪 & & \\
\hline $\mid$ & 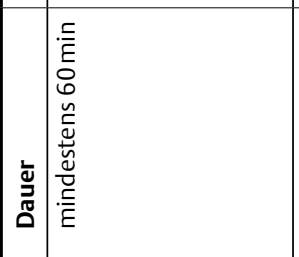 & 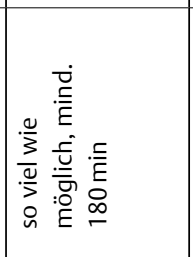 & 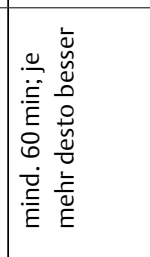 & 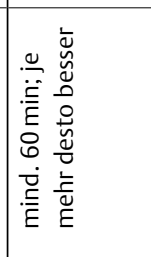 & 訄 & 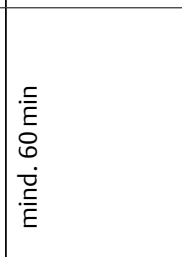 & 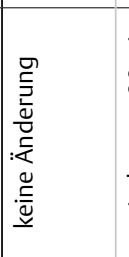 & 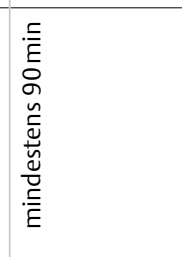 & \\
\hline 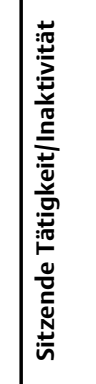 & I & 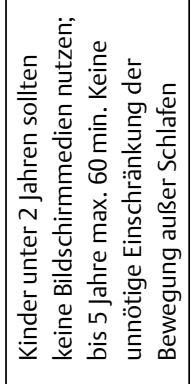 & 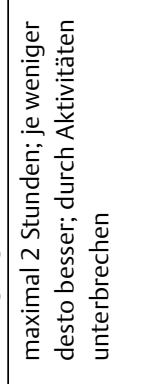 & 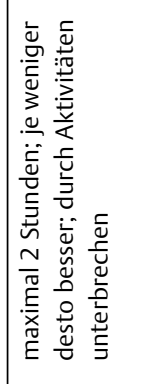 & 1 & & & 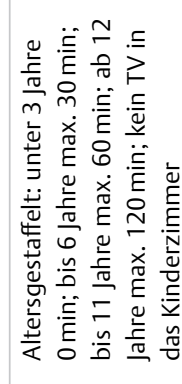 & \\
\hline 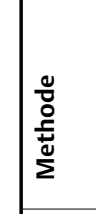 & ' & & & & 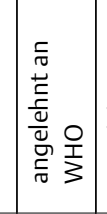 & 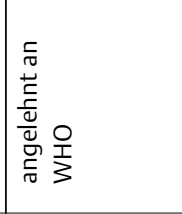 & & 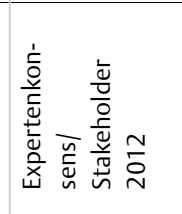 & 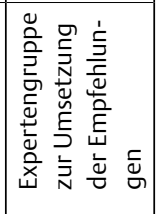 \\
\hline 贺 & 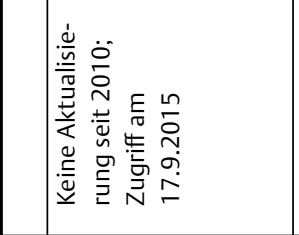 & & & & 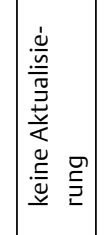 & & 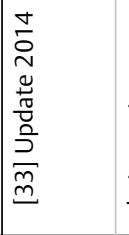 & 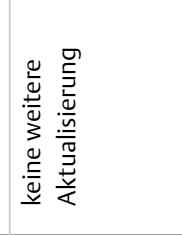 & \\
\hline $\mid$ & $\underline{\underline{\underline{S}}}$ & 槿 & 槿 & 槿 & $\overline{\underline{\tilde{m}}}$ & $\overline{\widetilde{\varrho}}$ & & $\Xi$ & 制 \\
\hline 䔅 & $\frac{0}{3}$ & 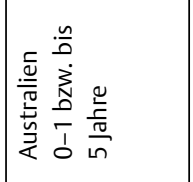 & 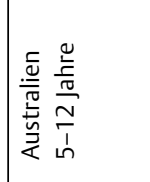 & 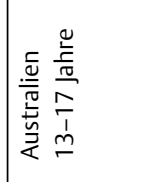 & 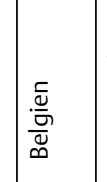 & 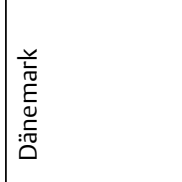 & | & 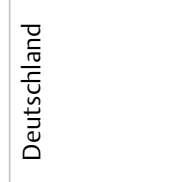 & 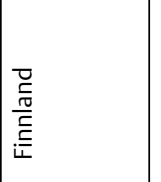 \\
\hline
\end{tabular}




\begin{tabular}{|c|c|c|c|c|c|c|}
\hline 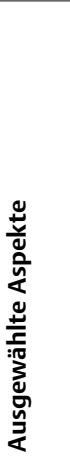 & 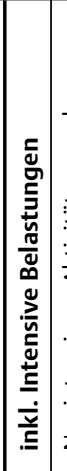 & 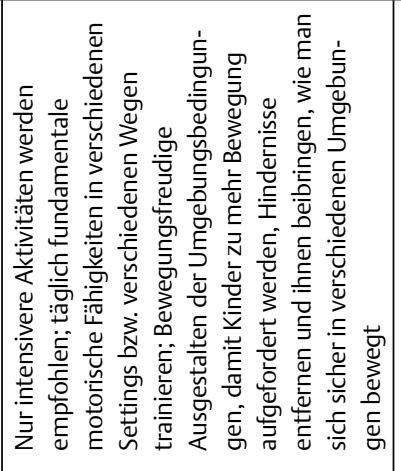 & 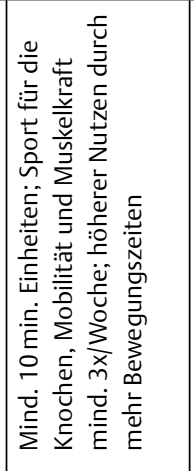 & 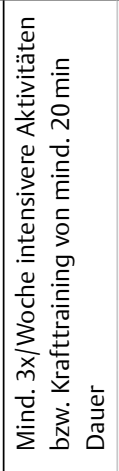 & 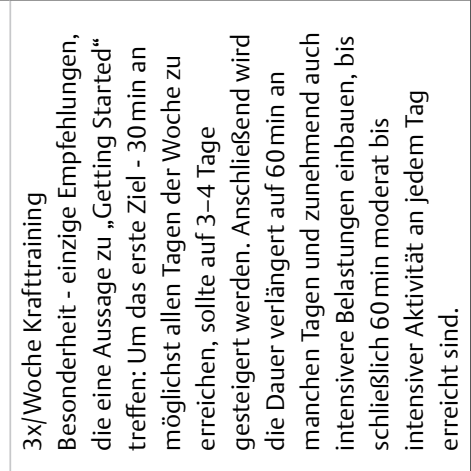 & 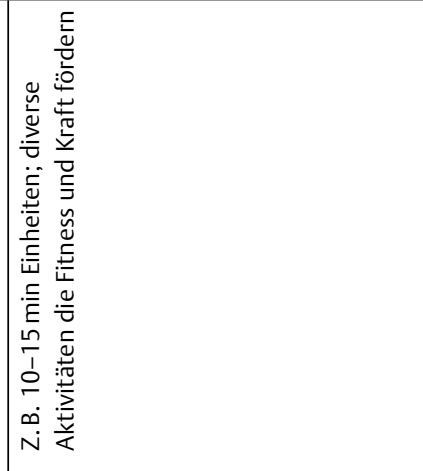 \\
\hline \multirow{3}{*}{ 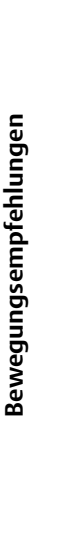 } & 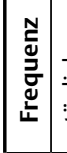 & 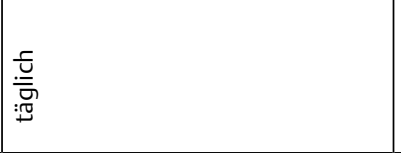 & $\begin{array}{l}\text { 点 } \\
\text { : } \\
: \mathbb{J}\end{array}$ & 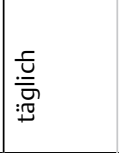 & 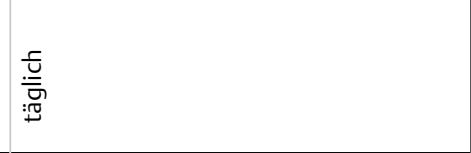 & 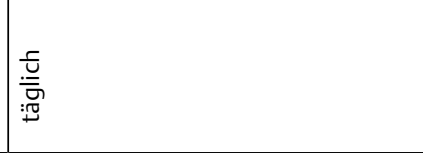 \\
\hline & 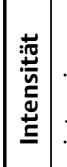 & 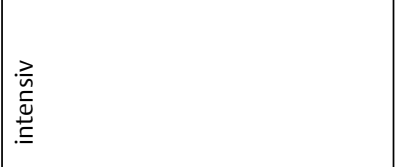 & 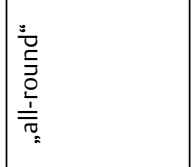 & 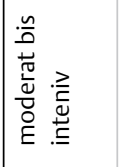 & 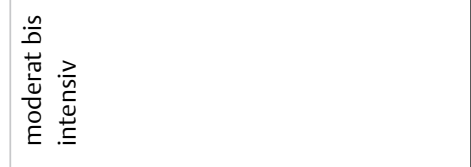 & \\
\hline & . & 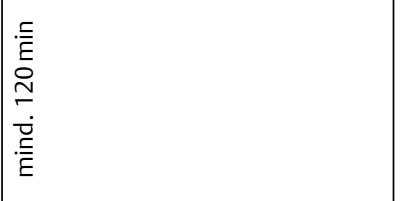 & 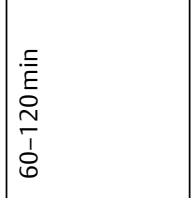 & 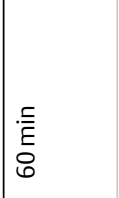 & 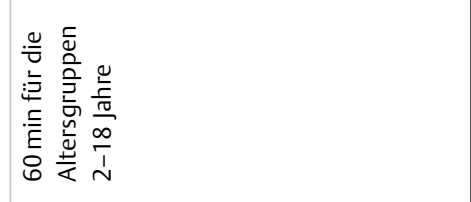 & 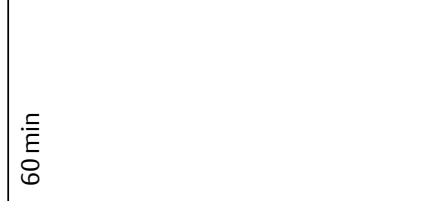 \\
\hline 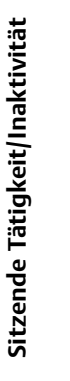 & & & 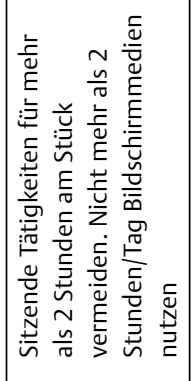 & & & \\
\hline 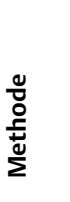 & & 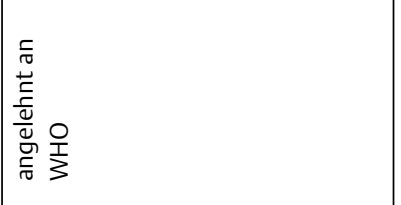 & 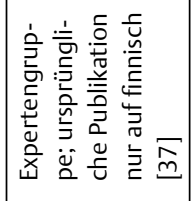 & 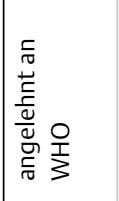 & 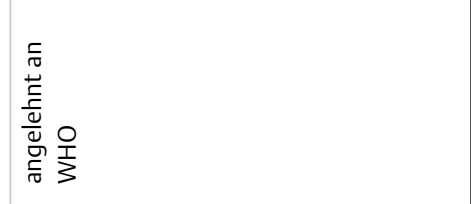 & 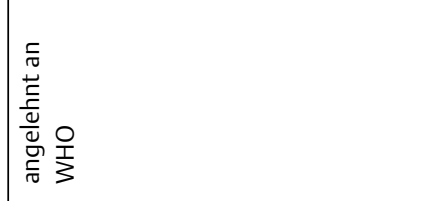 \\
\hline 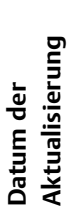 & & & & 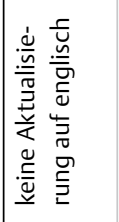 & 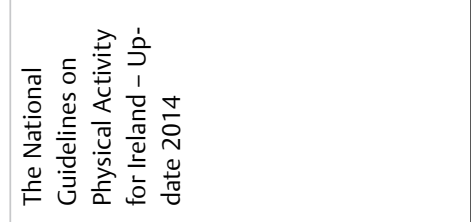 & 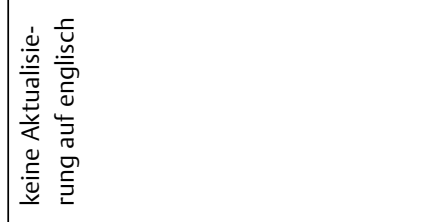 \\
\hline$\frac{N}{\tilde{u}}$ & & $\stackrel{\bar{n}}{\stackrel{n}{m}}$ & $\begin{array}{l}\tilde{D} \\
\stackrel{m}{2}\end{array}$ & $\stackrel{\infty}{m}$ & $\underset{\Xi}{\mathbb{\Xi}}$ & 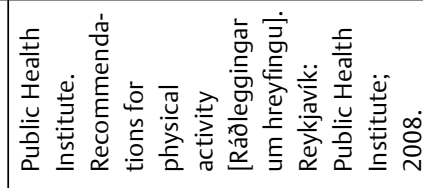 \\
\hline ర్త & & 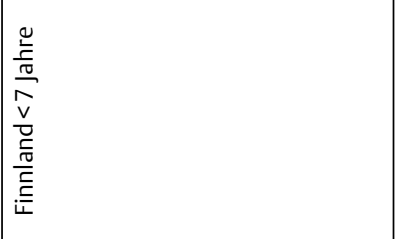 & 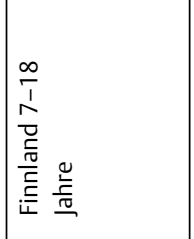 & 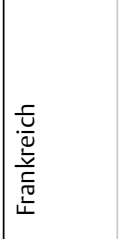 & 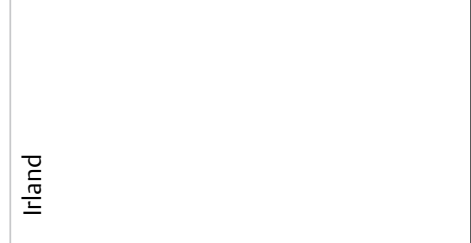 & 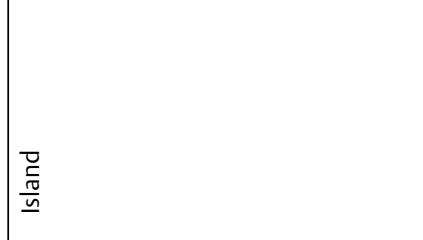 \\
\hline
\end{tabular}




\begin{tabular}{|c|c|c|c|c|c|c|c|c|c|}
\hline 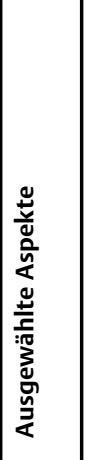 & | & & 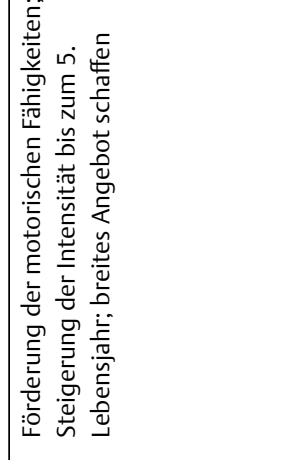 & 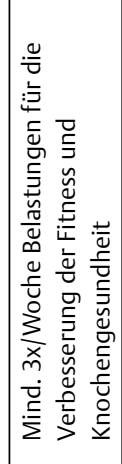 & 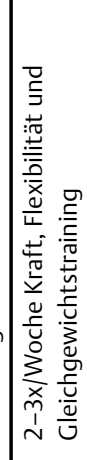 & 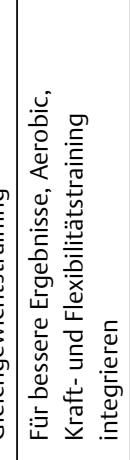 & 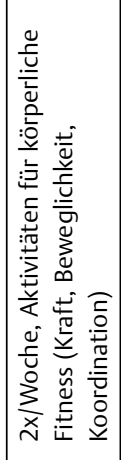 & 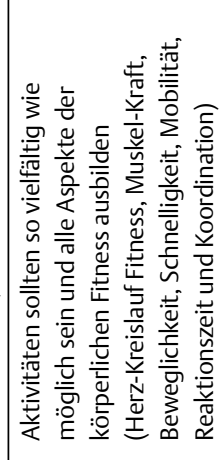 & 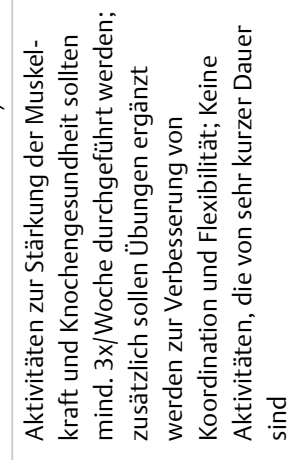 \\
\hline \multirow{3}{*}{ 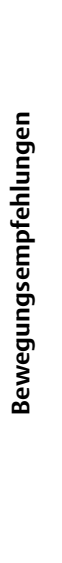 } & 竞 & & $\begin{array}{l}\text { 站 } \\
\text { : } \\
\end{array}$ & 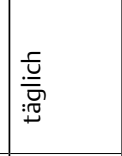 & 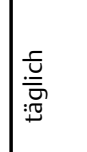 & 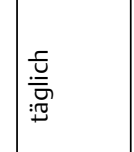 & 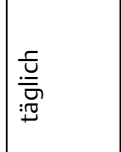 & \begin{tabular}{|l}
$\frac{5}{5}$ \\
竘 \\
\end{tabular} & 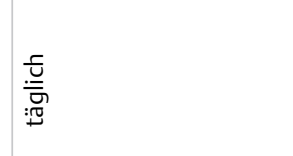 \\
\hline & : & & & 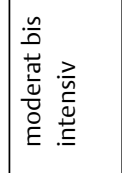 & 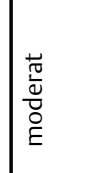 & 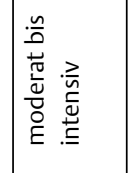 & 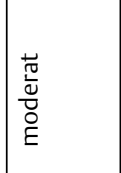 & 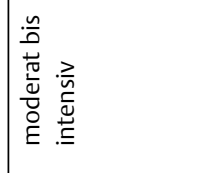 & 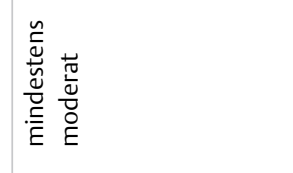 \\
\hline & 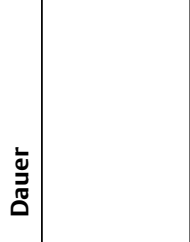 & & 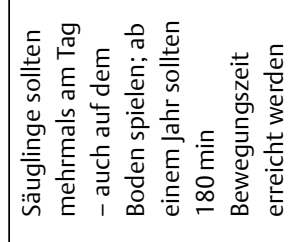 & 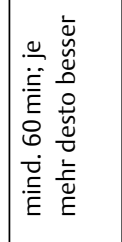 & 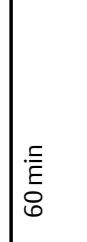 & 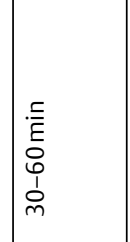 & 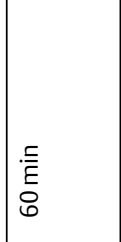 & 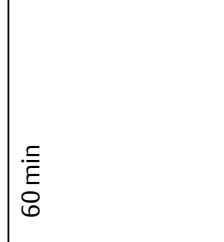 & \begin{tabular}{|l} 
高 \\
蒠
\end{tabular} \\
\hline 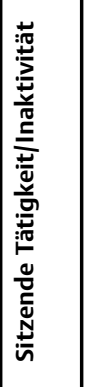 & 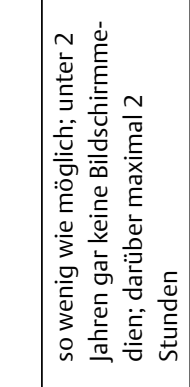 & 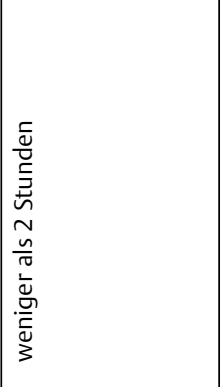 & & & & 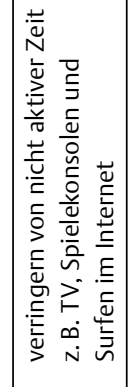 & & & 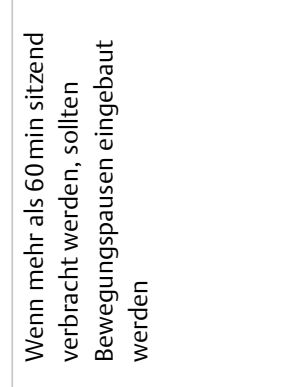 \\
\hline 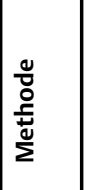 & & & & & 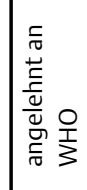 & 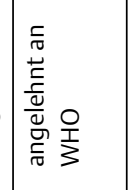 & 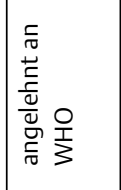 & 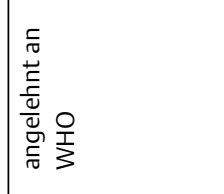 & 1 \\
\hline 焉 & & & & & 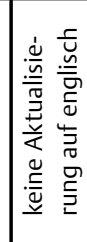 & & & 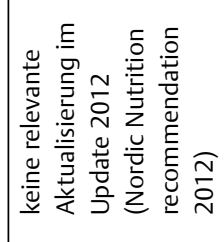 & $\bar{\rho}$ \\
\hline 芯 & $\Phi$ & $\underset{\mathcal{I}}{\bar{d}}$ & 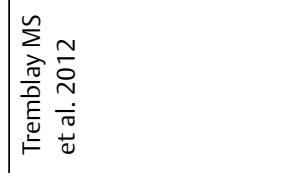 & $\Phi$ & 命 & 守 & $\bar{\Xi}$ & $\bar{\Xi}$ & হ \\
\hline 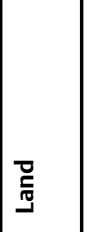 & 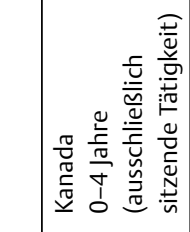 & 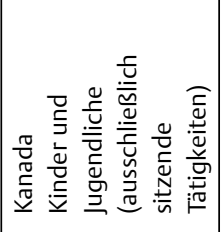 & 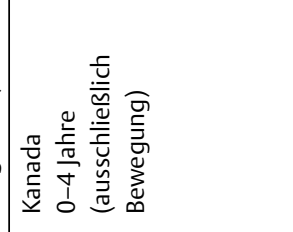 & 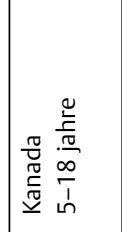 & 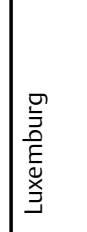 & $\frac{\frac{\pi}{\frac{\pi}{N}}}{2}$ & 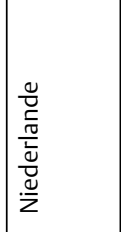 & 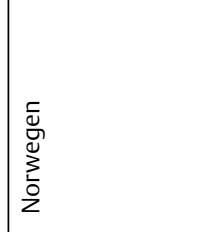 & 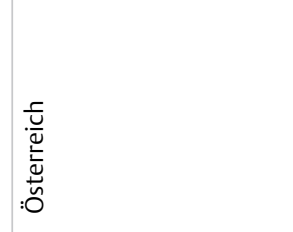 \\
\hline
\end{tabular}




\begin{tabular}{|c|c|c|c|c|c|c|c|c|c|}
\hline 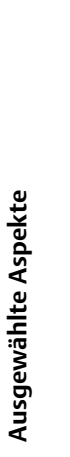 & 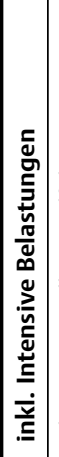 & 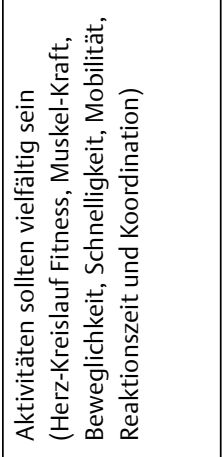 & 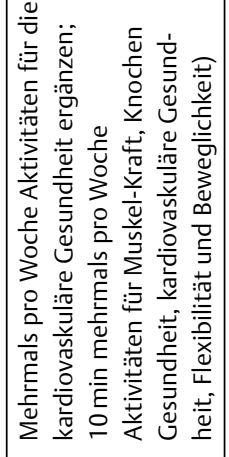 & 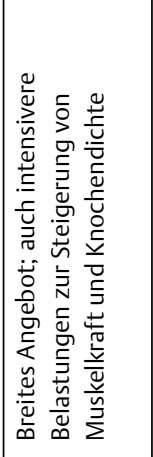 & & 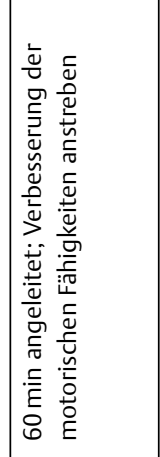 & 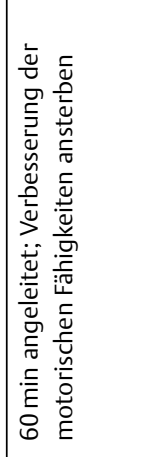 & 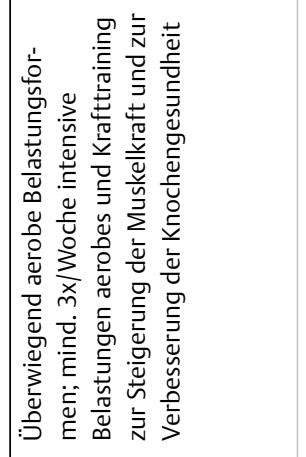 & 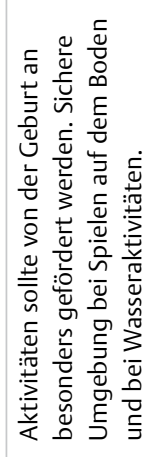 \\
\hline \multirow{3}{*}{ 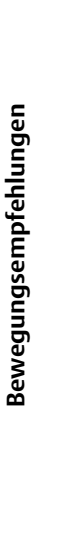 } & 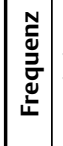 & 站 & 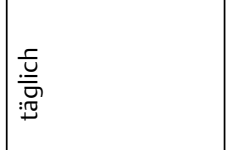 & 点 & 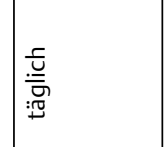 & $\begin{array}{l}\frac{\underline{\underline{5}}}{\underline{\underline{T}}} \\
: \underline{7}\end{array}$ & 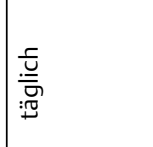 & 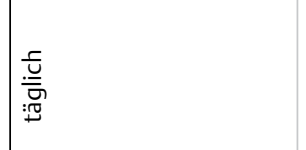 & \\
\hline & 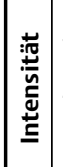 & 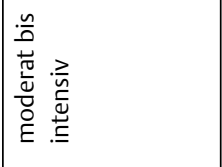 & 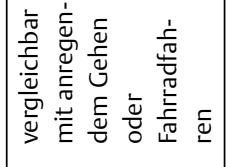 & 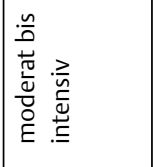 & 1 & 1 & 1 & 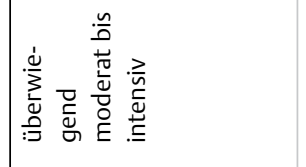 & \\
\hline & $\begin{array}{l}\overline{\mathrm{J}} \\
\bar{\Xi} \\
\mathrm{\Xi}\end{array}$ & 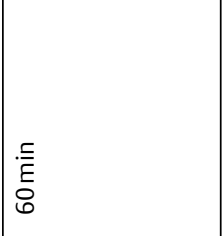 & 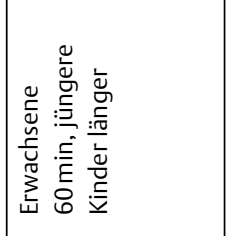 & 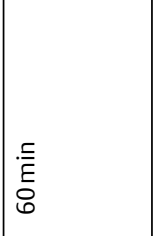 & 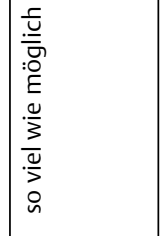 & 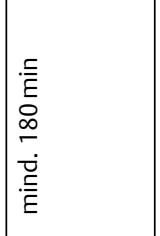 & 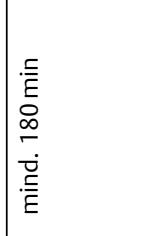 & 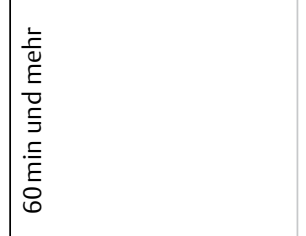 & \\
\hline 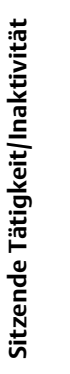 & & & 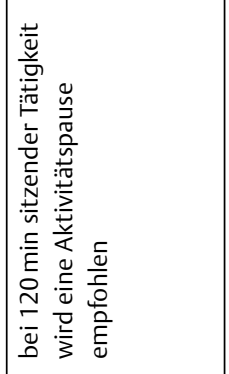 & & 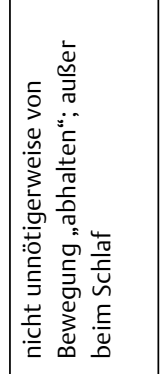 & 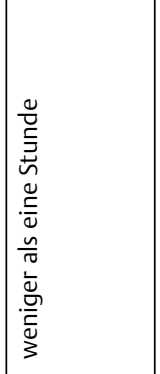 & 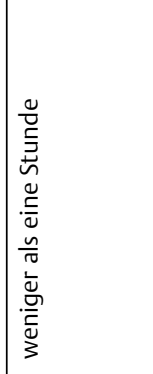 & 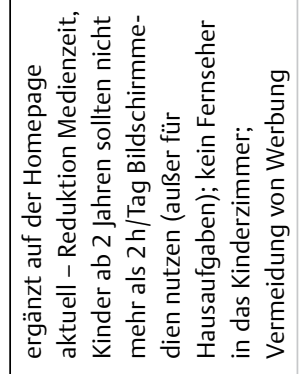 & 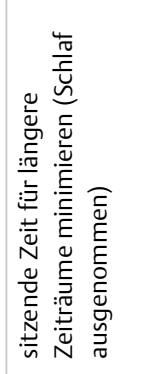 \\
\hline 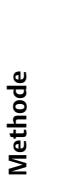 & & 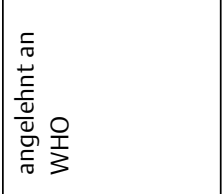 & 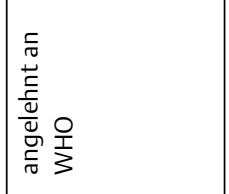 & 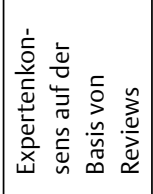 & & & & 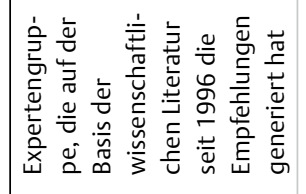 & \\
\hline 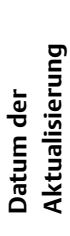 & & 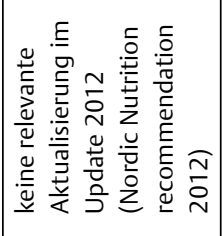 & & & 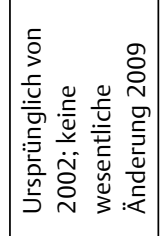 & 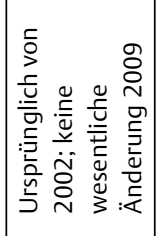 & 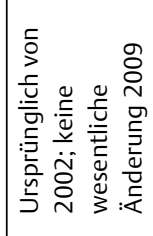 & 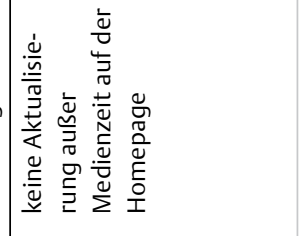 & \\
\hline 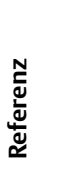 & & $\stackrel{\mathscr{I}}{\Xi}$ & $\bar{q}$ & $\underline{\underline{n}}$ & $\underline{\sigma}$ & $\underline{\sigma}$ & $\sigma$ & 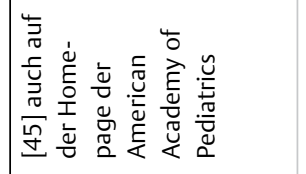 & 寻 \\
\hline 岢 & & 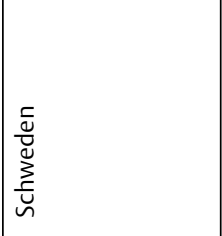 & $\begin{array}{l}\cdot \stackrel{N}{N} \\
\frac{\sum_{\tilde{N}}}{\tilde{n}}\end{array}$ & 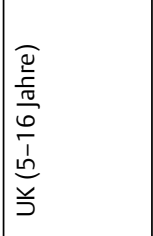 & 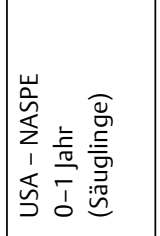 & 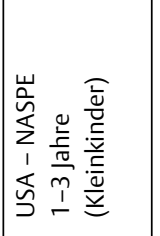 & 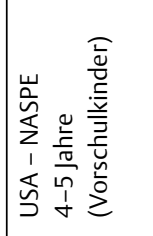 & 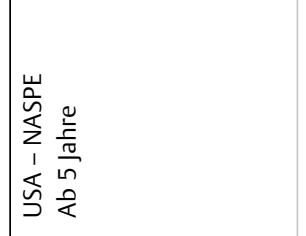 & 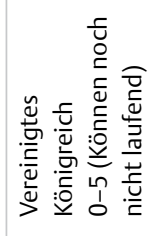 \\
\hline
\end{tabular}




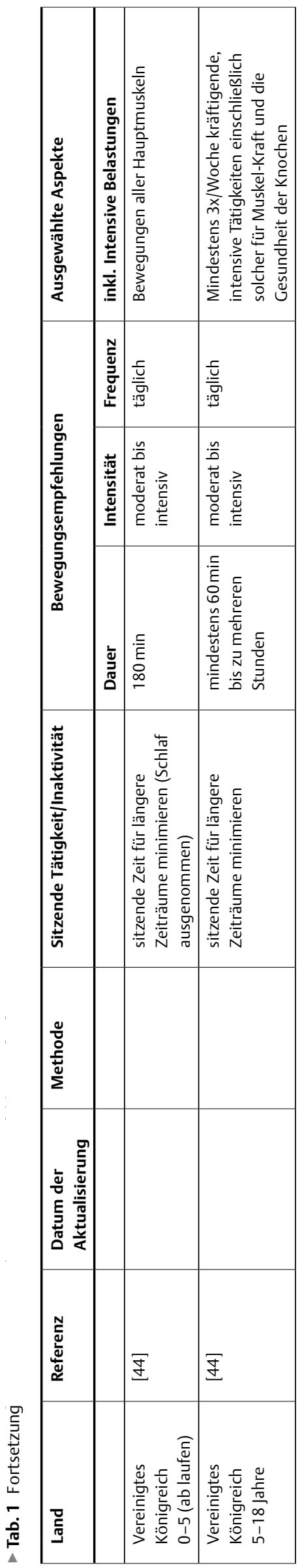

wicklung verbunden ist. Über das genaue Ausmaß, die Intensität, Häufigkeit und/oder Bewegungsform/Sportart lassen sich allerdings (noch) keine eindeutig begründeten Aussagen treffen. Konsens besteht aber in allen bisher für diese Altersgruppe vorliegenden Empfehlungen, so viel Bewegung wie möglich anzubieten bzw. den natürlichen Bewegungsdrang nicht einzuschränken und die Eltern bzw. Betreuungspersonen für die Bedeutung von Bewegung zu sensibilisieren [2,8]. So gibt es Hinweise, dass das Ausmaß an körperlicher Aktivität im Vorschulalter die Bewegungszeit im Erwachsenenalter positiv beeinflusst [16]. Gefährdungen durch eine vermehrte Bewegung wurden nicht beschrieben [2], die Umgebungsbedingungen sollten entsprechend kindgerecht sicher sein. Zusätzlich wird auf angeleitete und nicht angeleitete Aktivität in der Altersgruppe bis unter 5 Jahre verwiesen; > Tab. 1.

\section{Grundschul- und Jugendalter}

Für die weiteren Altersgruppen, insbesondere ab dem Grundschulalter ist die Datenlage deutlich besser. Als Grundlage für die kanadischen Empfehlungen untersuchten Janssen u. LeBlanc [3] den Benefit von körperlicher Aktivität für Schulkinder und fassten folgende Auswirkungen zusammen: Mit dem Einfluss auf Cholesterin und die Blutlipide beschäftigten sich 9 Studien. In einer Beobachtungsstudie zeigte sich, dass körperlich weniger leistungsfähige 12- bis 19-jährige Mädchen ein etwa 1,9-fach höheres Risiko einer Hypercholesterinämie aufwiesen, Jungen ein etwa 3,7-fach höheres. Die übrigen experimentellen Studien beschäftigten sich mit erhöhten Blutfettwerten und/oder Adipositas. Die Ergebnisse waren inkonsistent. Aerobe Belastungen führten zu einer Verbesserung v. a. der Triglyzeridspiegel. Die Effekte von Krafttraining auf die Lipidspiegel waren minimal. Mit arterieller Hypertonie befassten sich 11 Studien. Die Effektstärken waren aber für das Ausdauertraining gering $(-1,39 \mathrm{mmHg}$ bezüglich des systolischen, $-0,39 \mathrm{mmHg}$ bezüglich des diastolischen Blutdrucks) und für das Krafttraining nicht wirklich beurteilbar. Die Zusammenhänge mit Übergewicht und Adipositas wurden deutlich häufiger untersucht. In 31 Studien, die integriert wurden, lag die Effektstärke für Ausdauertraining bei - 0,40 für den prozentualen Körperfettanteil und - 0,07 für den BMI, für Krafttraining lag die Effektstärke bei - 0,19 für den prozentualen Körperfettanteil. In Untersuchungen über Zusammenhänge mit dem metabolischen Syndrom bzw. dem Nüchterninsulinspiegel zeigen sich Effektstärken für das Ausdauertraining in der Höhe von - 0,60 bzw. für das Krafttraining von - 0,31. Positive Ergebnisse wurden auch für die Knochendichte, das Auftreten von Verletzungen bzw. Depressionen und entsprechenden Symptomen berichtet.

Wegweisende Differenzierungen nach weiteren Einflussgrößen wie z. B. Bewegungsformen/Sportarten, Intensität, soziale und kulturelle Voraussetzungen, Alter und Geschlecht fehlen nach wie vor. In einem aktuelleren Review wurde der Zusammenhang zwischen der muskulären Fitness als „Outcome“ der körperlichen Aktivität und der Reduktion von (zentraler) Adipositas, kardio-metabolischen Risikofaktoren wie z. B. Insulinresistenz, Blutdruck, Knochengesundheit sowie Selbstwertgefühl untersucht [17]; je höher die Umfänge von körperlicher Aktivität sind, desto höher wird auch der gesundheitliche Nutzen angenommen. Für Deutschland zeigt sich anhand des MoMo-Kollektivs (MoMo: Motorik Modul als Subgruppe der repräsentativen KiGGS-Studie), dass unter Berücksichtigung 
\ab. 2 Empfehlungen für körperliche Aktivität und Inaktivität im Kindes- und Jugendalter.

\begin{tabular}{|c|c|}
\hline \multicolumn{2}{|c|}{ Säuglinge und Kleinkinder } \\
\hline & $\begin{array}{l}\text { Säuglinge und Kleinkinder sollten so wenig wie möglich in ihrem natürlichen Bewegungsdrang gehindert werden und sich so viel wie möglich } \\
\text { bewegen; auf sichere Umgebungsbedingungen ist zu achten. }\end{array}$ \\
\hline \multicolumn{2}{|c|}{ Kindergartenkinder (4-6 Jahre) } \\
\hline & Für Kindergartenkinder soll eine angeleitete und nichtangeleitete Bewegungszeit von $180 \mathrm{~min} /$ Tag und mehr erreicht werden. \\
\hline \multicolumn{2}{|r|}{ Grundschulkinder (6-11 Jahre) } \\
\hline & $\begin{array}{l}\text { Für Kinder ab dem Grundschulalter soll eine tägliche Bewegungszeit von } 90 \text { min und mehr mit moderater bis intensiver Intensität erreicht werden. } \\
60 \text { min davon können durch Alltagsaktivitäten, z. B. Schulweg, jedoch mindestens } 12000 \text { Schritte/Tag absolviert werden. }\end{array}$ \\
\hline \multicolumn{2}{|r|}{ Jugendliche (12-18 Jahre) } \\
\hline & $\begin{array}{l}\text { Für Jugendliche soll eine tägliche Bewegungszeit von } 90 \text { min und mehr mit moderater bis intensiver Intensität erreicht werden. } 60 \text { min davon } \\
\text { können durch Alltagsaktivitäten, z. B. mindestens } 12000 \text { Schritte/Tag absolviert werden. }\end{array}$ \\
\hline \multicolumn{2}{|c|}{ Spezifische Aspekte } \\
\hline & $\begin{array}{l}\text { Besonderheiten, aber auch Neigungen, Bedürfnisse und mögliche Barrieren der jeweiligen Zielgruppe, z. B. Alter, Geschlecht, soziokulturelle } \\
\text { Faktoren, sollen berücksichtigt werden. }\end{array}$ \\
\hline & Allgemein soll eine Förderung der motorischen Leistungsfähigkeit alters- und geschlechtsangepasst werden. \\
\hline & $\begin{array}{l}\text { Ab dem Grundschulalter soll zur Verbesserung von Kraft und Ausdauer an 2-3 Tagen pro Woche intensive Beanspruchung der großen Muskelgrup- } \\
\text { pen erfolgen, jeweils unter Berücksichtigung des jeweiligen Entwicklungsstandes. }\end{array}$ \\
\hline & $\begin{array}{l}\text { Bewegungsarme Kinder und Jugendliche sollten schrittweise an das Ziel herangeführt werden, z. B. durch zunächst } 30 \text { min Bewegung an 1-2 Tagen } \\
\text { pro Woche. Anschließend werden der zeitliche Umgang, dann die Intensität gesteigert. }\end{array}$ \\
\hline \multicolumn{2}{|r|}{ Sitzende Tätigkeiten in der Freizeit/Bildschirmmedien } \\
\hline \multirow{2}{*}{\multicolumn{2}{|c|}{$\begin{array}{l}\text { Vermeidbare Sitzzeiten sollten auf ein Minimum reduziert werden. Neben (motorisiertem) Transport, z. B. in Babyschale oder Kindersitz oder unnötig im } \\
\text { Haus verbrachten Zeiten, betrifft dies insbesondere die Reduktion des Bildschirmmedienkonsums auf ein Minimum: } \\
\text { - Säuglinge und Kleinkinder: } 0 \text { min } \\
\text { - Kindergartenkinder: möglichst wenig, maximal } 30 \mathrm{~min} / \text { Tag } \\
\text { - Grundschulkinder: möglichst wenig, maximal } 60 \mathrm{~min} / \mathrm{Tag} \\
\text { - Jugendliche: möglichst wenig, max. } 120 \mathrm{~min} / \mathrm{Tag}\end{array}$}} \\
\hline & \\
\hline & $\begin{array}{l}\text { derate Intensität entspricht einer leichten Steigerung der Herzfrequenz bzw. etwas angeregterer Atmung (s.a. http://www.cdc.gov/ } \\
\text { icalactivity/basics/children/); }{ }^{2} \text { Intensive Intensität entspricht einer deutlichen Steigerung der Herzfrequenz bzw. erheblich angeregteren Atmung } \\
: / / \text { www.cdc.gov/physicalactivity/basics/children) }\end{array}$ \\
\hline
\end{tabular}

der Aktivität in der Freizeit, wie auch in Institutionen (Verein, Schule/Kindergarten usw.) eine wöchentliche Bewegungszeit der Jungen von durchschnittlich etwa 480 min und bei Mädchen von etwa $400 \mathrm{~min}$ ( $n=4401$, [18]). Auch wenn es sich bei dieser Betrachtung lediglich um die Summation der angegebenen Mittelwerte handelt, und dies nicht die Realität ausgewählter Subgruppen wiederspiegelt, kann vorsichtig abgeleitet werden, dass durchschnittlich auf einen Tag bezogen voraussichtlich mehr als 60 min an täglicher Bewegungszeit erreicht werden. Diese aktuell meist als Minimum geltenden 60 min basieren auf Expertenkonsens bzw. epidemiologischen Daten, wie viel bzw. wenig Bewegung absolviert wird, und weniger auf einem daraus ableitbarem gesundheitlichen Nutzen. Daher werden inzwischen teils höhere Bewegungszeiten, z. B. Finnland, oder zumindest für ausgewählte Altersgruppen, z. B. England, USA, empfohlen, weil damit ausdrücklich ein größerer gesundheitlicher Benefit verbunden wird ( $\triangleright$ Tab. 1). Entsprechend wurde bereits 2012 im Expertenkonsens in Deutschland für die Altersgruppe ab dem Grundschulalter eine tägliche Bewegungszeit von 90 min für Deutschland empfohlen [1,4].

\section{Alltagsaktivitäten bzw. Nutzen verschiedener Bewegungsformen}

Über den Nutzen von körperlicher Aktivität beim die aktive Ausgestaltung von Transportwegen gibt es bislang nur wenige Studien; in Anlehnung an die Befunde im Erwachsenenalter wird im Deut- schen Expertenkonsens der Nutzen/Einsatz von Alltagsaktivitäten, d. h. Schulweg, empfohlen $[1,10,19]$. In der Literatur finden sich Hinweise zur Übersetzung der Empfehlung von 60 min Bewegung am Tag in etwa 11500 Schritte/Tag [19] bzw. dezidierter nach Alter und Geschlecht für Mädchen im Grundschulalter zwischen 10000 bis 12000 Schritte/Tag bzw. Jungen dieser Altersstufe zwischen 13000 bis $15000 /$ Tag bzw. für in der späten Kindheit und im Jugendalter zwischen 11000 und 11700 [10].

In verschiedenen Empfehlungen werden Hinweise zu bestimmten Formen körperlicher Aktivität gegeben. Diese richten sich aber im Wesentlichen an Eltern und Betreuungspersonen und basieren nicht auf einer wissenschaftlichen Grundlage. Bei jüngeren Kindern ist dies das Spielen auf dem Fußboden und Toben, bei älteren z. B. das Radfahren. Allerdings liegen keine Studien für die Bevorzugung oder Überlegenheit einzelner Bewegungsformen oder Sportarten vor. Grundtenor ist, den Neigungen des Kindes zu folgen und die Bewegungszeit, v. a. bei jüngeren Kleinkindern und Kindergartenkindern, nicht zu begrenzen. Für ältere Kinder werden in den meisten Empfehlungen Angaben zu Bewegungsformen gemacht, die Ausdauer bzw. Muskelkraft verbessern sollen. Dabei wird an 3 und mehr Tagen pro Woche eine angemessene Beanspruchung der großen Muskelgruppen zur Verbesserung von Muskelkraft, Knochendichte und kardiorespiratorischen Fitness empfohlen. Konkrete Angaben zu Intensitäten werden nicht gegeben, allerdings muss stets der individuelle Entwicklungsstand berücksichtigt werden (u. a. [5, 7]; s.a. > Tab. 1). 


\section{Mögliche Risiken}

Die Bewegungsausführungen sollten altersentsprechend durchgeführt werden, um keine Schäden am wachsenden Organismus herbeizuführen und vor allem den Kindern und Jugendlichen Spaß und Freude machen [14]. Für jüngere Kinder (unter 1 bzw. unter 3 Jahren) wird zusätzlich empfohlen, die Umgebungsbedingungen sicher zu gestalten. In einem aktuellen Review wurde die Verletzungsrate aus 8 integrierten Studien für die Altersgruppe 8-12 Jahre zusammengefasst [20]. Insgesamt lag die Zahl an Unfällen, die medizinisch versorgt werden mussten, etwa zwischen 0,2 und 0,3 Ereignissen in 1000 Aktivitätsstunden. Die absolute Zahl war in nicht-organisierten Bewegungsfeldern höher als in organisierten Sportangeboten. Generell werden Differenzierungen in verschiedene Altersstufen, v. a. im Bereich der Vorschulkinder und jünger bislang nur in UK, USA, Kanada und Australien getroffen, die jedoch alle auf den gleichen Quellen beruhen. Irland umfasst in seinen Empfehlungen die Altersgruppe von 2 bis 18 Jahren. Bei Jugendlichen konnte dagegen gezeigt werden, dass durch ein gezieltes Training (v. a. der Koordination und Kraft) Sportverletzungen vorgebeugt werden kann [21].

Bei inaktiven Kindern und Jugendlichen wird ausschließlich in den irischen Empfehlungen ein schrittweises Vorgehen gefordert: Um das erste Ziel - 30 min an möglichst allen Tagen der Woche zu erreichen, sollte die Bewegungszeit auf 3-4 Tage gesteigert werden. Anschließend wird die Dauer verlängert auf 60 min an manchen Tagen und zunehmend sollten auch intensivere Belastungen eingebaut werden, bis schließlich 60 min moderater bis intensiver Aktivität an jedem Tag erreicht sind ([22] sowie update 2014; - Tab. 1). In ausgewählten Empfehlungen werden auch kleinere Einheiten von bis zu 15 min Dauer aufgeführt (z. B. Island und Deutschland).

\section{Sitzende Tätigkeiten in der Freizeit/Nutzung von Bildschirmmedien}

Zunehmend hat sich „sedentary behaviour“ bzw. Sedentarismus als eigenständiger Risikofaktor für die Entwicklung von Übergewicht, aber auch für psychosoziale Auffälligkeiten herausgestellt [23]. Es liegt bislang keine einheitliche Definition vor; im Rahmen der kanadischen Empfehlungen wird folgende Definition genutzt [23]:

- als „sedentary“ gelten „Aktivitäten“, wie Sitzen, Fernsehschauen, Videospiele spielen usw., die durch wenig Bewegung bzw. einen geringen Energieverbrauch unter 1,5 MET gekennzeichnet sind.

- Sedentarismus wird als überwiegend „sedentary behaviour“ definiert, das durch ein Minimum an Bewegung mit geringen Energieverbrauch unter 1,5 MET gekennzeichnet ist. Neben Hinweisen auf eine Limitierung von (motorisiertem) Transport im Sitzen, z. B. in Babyschale oder Kindersitz sowie Zeiten, die unnötig im Haus verbracht werden, beziehen sich die Empfehlungen im Wesentlichen auf die Nutzung audiovisueller Medien in der Freizeit; z. B. Videospiele und TV-Konsum, die explizit nicht im schulischen Kontext genutzt werden [23]. In einem entsprechenden systematischen Review, das wiederum die Grundlage für die kanadischen Empfehlungen darstellte, wurde eine höhere Bildschirmmedienzeit (meist 2 bzw. 3 Stunden) mit einer gesteigerten Prävalenz von Übergewicht und Adipositas, einem höheren Körperfettanteil, einer geringeren Fitness, weniger Selbstbewusstsein, schlechterer akademischer Leistung bzw. mehr Verhaltensauffälligkeiten in Verbindung gebracht [24]. Diesbezüglich zeigt sich allerdings im negativen - eine Dosis-Wirkungsbeziehung [24]. Unter 2 Jahren wird keine Bildschirmmedienzeit empfohlen [25]. In diesem Kontext wird auch der Hinweise für Eltern gegeben, Kinder nicht dem Bildschirmmedienkonsum auszusetzen, d. h. Fernseher nicht nebenbei laufen zu lassen und vor allem nicht im Kinderzimmer einen Fernseher aufzustellen. Für die Gruppen der 1-bis 4-jährigen [26] bzw. 2- bis 5-jährigen [27] wird ein Maximum von einer Stunde Bildschirmzeit täglich empfohlen; stets allerdings mit dem Hinweis, dies aufgrund der negativen Folgen auf ein Minimum zu reduzieren. Im Deutschen Expertenkonsens wurde daher die Zeit der unnötigen Bildschirmmediennutzung auf maximal $30 \mathrm{~min} / \mathrm{Tag}$ im Kindergartenalter und maximal $60 \mathrm{~min} / \mathrm{Tag}$ im Grundschulalter festgelegt [1]. In höherem Alter werden meist 2 Stunden Bildschirmmedienzeit in der Freizeit angegeben [28]. Diese wurden im Deutschen Expertenkonsens ebenfalls für das Jugendalter übernommen [1].

\section{ZUSAMMENFASSUNG}

Zusammengefasst liegen nun erstmals dezidierte Empfehlungen bzgl. körperlicher Aktivität und Inaktivität für verschiedene Altersabschnitte im Kindes- und Jugendalter vor. Sicherlich ist der gesundheitliche Nutzen, v. a. für jüngere Kinder, im Wesentlichen auf Analogieschlüsse zurückzuführen, denn die Datenlage ist teilweise sehr spärlich. Außerdem wurden viele Empfehlungen auf Basis von Expertenmeinungen erstellt, nur selten wurden methodisch feststehende Leitlinienverfahren (z. B. AGREE in Kanada [1]) genutzt. Ein weiteres Problem ist die Orientierung an der Epidemiologie und nicht an dem tatsächlichen Bedarf bzw. der Dosis an Bewegungszeit. Das bedeutet, dass die Frage, wie umfangreich und intensiv müssen Aktivitäten für welcher Altersstufe, ggf. unterschieden nach Geschlecht, ausgestaltet sein, um tatsächlich eine gesunde Entwicklung zu garantieren bzw. diese zu fördern, aktuell nicht beantwortet werden kann. Generell sind aber Aussagen über die Evidenz und Empfehlungen im Gesundheitswesen bzw. Bewegungsbereich komplex; so ist es - auch unter Berücksichtigung der jungen Altersstufen fraglich, welche Endpunkte adäquat und relevant sind, welche Evidenz für jeden dieser Endpunkte betrachtet bzw. wie die Qualität bewertet werden soll. Die bisherige Datenlage und mögliche, gut begründete Analogieschlüsse unterstreichen jedoch die generelle Bedeutung von (möglichst viel von) körperlicher Bewegung bzw. der Begrenzung vermeidbarer Inaktivitäten. Inwiefern sich diese Empfehlungen in der Realität in verschiedenen Settings und diversen Zielgruppen umsetzen, bleibt wiederum eine weitere Herausforderung.

Interessenkonflikt

Die Autoren geben an, dass kein Interessenkonflikt besteht. 